Classification

Physics Abstracts

64.70D -82.70

\title{
Polydispersity and the fluid-crystalline phase transition
}

\author{
E. Dickinson and R. Parker \\ Procter Department of Food Science, University of Leeds, Leeds LS2 9JT, U.K.
}

(Reçu le 15 octobre 1984, accepté le 17 janvier 1985)

\begin{abstract}
Résumé. - On part du postulat d'un degré "critique " de polydispersité au-delà duquel les états liquides et cristallins se confondent. Dans le cas d'un système avec une distribution triangulaire de la taille des particules, un calcul permet d'estimer que la disparition de la transition de fusion se produira à des niveaux de polydispersité légèrement au-dessous de $30 \%$.
\end{abstract}

\begin{abstract}
A « critical » degree of polydispersity is postulated beyond which liquid and crystalline states merge. For a system with a triangular particle-size distribution, the disappearance of the melting transition is estimated to occur at levels of just less than $30 \%$ polydispersity.
\end{abstract}

Freezing occurs at a first-order phase transition when the free energy of an ordered state(crystal) becomes lower than the free energy of a disordered state(liquid). On the solid side of the transition, the thermodynamically unfavourable change in configurational entropy is more than offset by the favourable change in total configurational energy, a situation that becomes optimized when all particles in the system are identical - as in a pure atomic or molecular material. In real colloids the particles are usually not identical, and the effect of polydispersity on the order-disorder transition is towards disruption of the crystalline state, since particles with radii much greater than the mean cannot easily be accommodated on a lattice at low potential energy without breaking down long-range order.

Theory shows $[1,2]$ that an increase in polydispersity has a large positive effect on the free energy of the colloidal crystalline phase, but a small negative effect on the free energy of the liquidlike phase. This can be readily understood for the case of mixing hard spheres of different sizes. With liquid-like states there is a small drop in non-combinatorial free energy associated with the negative volume change on mixing of hard spheres at constant pressure. With solid-like, nonglassy states, however, the free energy of the mixture is much greater than that of the unmixed hard spheres due mainly to the fact that smaller spheres must occupy « cells " with unfavourably large « free volumes " so as to maintain long-range order without particle overlap.

For a model system of spherical colloidal particles, simulation data show $[3,4]$ that the orderdisorder transition region narrows with increasing polydispersity. An obvious inference is that the freezing transition disappears altogether above a certain " critical " degree of polydispersity, so that only glassy states are observed at high density. The exact position of any merging of fluid and crystalline states will undoubtedly depend on the shape of the particle-size distribution and the nature of the interparticle forces. That being said, it seems reasonable to expect similar behaviour for all systems of strongly repulsive particles whose sizes are distributed symmetrically about the 
mean. It therefore seems worthwhile to estimate a " critical » degree of polydispersity from the simulation data obtained previously [4].

Consider an assembly of spherical particles with radii distributed according to the triangular function

$$
P(a)=[(\delta \bar{a})-|a-\bar{a}|] /(\delta \bar{a})^{2}, \quad(1-\delta \leqslant a / \bar{a} \leqslant 1+\delta)
$$

where $P(a) \mathrm{d} a$ is the probability of finding a particle with radius lying between $a$ and $a+\mathrm{d} a$, $\bar{a}$ is the most probable radius, and $\delta$ is the degree of polydispersity. We assume that particles interact with pairwise-additive colloid-type potentials of the form

$$
u\left(r_{i j}\right)=u_{\mathrm{R}}\left(r_{i j}\right)+u_{\mathrm{A}}\left(r_{i j}\right)
$$

where $r_{i j}$ is the distance between particle centres $i$ and $j$. The repulsion $u_{\mathrm{R}}\left(r_{i j}\right)$ has the screened Coulombic form

$$
u_{\mathrm{R}}\left(r_{i j}\right)=4 \pi \varepsilon \psi^{2}\left[a_{i} a_{j} /\left(a_{i}+a_{j}\right)\right] \ln \left[1+\exp \left(-\kappa s_{i j}\right)\right],
$$

where $s_{i j}=r_{i j}-\left(a_{i}+a_{j}\right), \varepsilon$ is the dielectric constant of the medium, $\psi$ is the particle surface potential, and $\kappa^{-1}$ is the double-layer thickness. The attraction $u_{\mathrm{A}}\left(r_{i j}\right)$ has the unretarded van der Waals form

$$
\begin{aligned}
u_{\mathrm{A}}\left(r_{i j}\right)=-(A / 6)\left\{2 a_{i} a_{j} /\left[r_{i j}^{2}-\left(a_{i}-a_{j}\right)^{2}\right]+\right. & 2 a_{i} a_{j} /\left[r_{i j}^{2}-\left(a_{i}+a_{j}\right)^{2}\right]+ \\
& \left.+\ln \left[r_{i j}^{2}-\left(a_{i}+a_{j}\right)^{2}\right] /\left[r_{i j}^{2}-\left(a_{i}-a_{j}\right)^{2}\right]\right\},
\end{aligned}
$$

where $A$ is Hamaker's constant. The potential parameters in equations (2) and (3) take the values $\psi=60 \mathrm{mV}, \varepsilon=\varepsilon_{0}$ (water), $\kappa^{-1}=30.8 \mathrm{~nm}$, and $A=2.5 \times 10^{-20} \mathrm{~J}$ (polystyrene in water). For the triangular distribution (Eq. (1)), the mean radius is

$$
\bar{a}=a_{\mathrm{m}}\left[1+\left(\delta^{2} / 2\right)\right]^{-1 / 3},
$$

where $a_{\mathrm{m}}$ is the equivalent monodisperse radius $(595 \mathrm{~nm})$. The product $\kappa a_{\mathrm{m}}$ is 19.5 which means that the Deryagin-type equation (Eq. (3)) is valid. The depth of the attractive potential well is small ( $\sim 0.11 k T)$, and so the pair interaction can be regarded as being essentially entirely repulsive, with particles $i$ and $j$ having an effective hard-sphere diameter only a few per cent greater than $a_{i}+a_{j}$

The equation of state of the model system of 108 particles has been determined by molecular dynamics simulation at densities in the vicinity of the freezing transition $[4,5]$. For the model system in its monodisperse form $(\delta=0)$, the computed volume change on melting, $10.0 \pm 0.5 \%$, is the same as that found for hard spheres [6]. This result demonstrates the considerable " hardness " of the chosen colloid potential at this electrolyte concentration $\left(0.1 \mathrm{~mol} \mathrm{~m}^{-3}\right)$ compared with softer-sphere potentials of the general form $r^{-n}$; with $n=12$, for example, the volume change on melting is only $3.8 \%[6]$.

In their simulations of the Lennard-Jones system, Streett et al. [7] found that state points mapped out an S-shaped curve in pressure-volume space; and they were able to locate the position of the order-disorder transition by means of a Maxwell « equal area " construction by analogy with the van der Waals loop in the gas-liquid régime. We adopt the same procedure here in analysing our colloid simulation data. That is, at each degree of polydispersity, the tie-line is located by the method of Streett et al. [7]. We acknowledge that the method is not rigorous, but it gives excellent agreement with the « exact " procedure of Hoover and Ree [8] for the monodisperse case $(\delta=0)$, and we see no reason why it should not be equally good for the polydisperse case. 


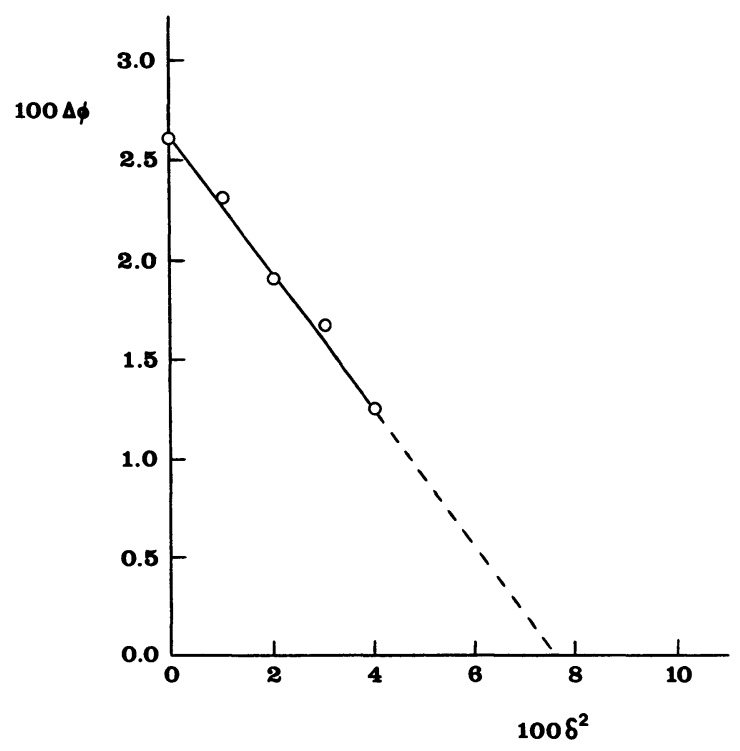

Fig. 1. - Effect of polydispersity on the volume change on melting. The change in volume fraction $\Delta \phi$ is plotted against the square of the degree of polydispersity $\delta^{2}$. The straight line extrapolates to $\delta^{2}=0.076$ at $\Delta \phi=0$.

Polydispersity reduces the volume change upon melting as indicated in figure 1 . The rationale for plotting the width of the transition, $\Delta \phi=\phi_{\text {solid }}-\phi_{\text {liquid, }}$, against $\delta^{2}$ comes from the observation $[3,4]$ that for $\delta \lesssim 0.3$ the compressibility factor of a polydisperse crystal increases strongly as $\delta^{2}$ whereas that for a polydisperse fluid is essentially independent of $\delta$ at constant particle volume fraction $\phi$. As $\delta$ increases, the melting point moves progressively to higher volume fractions, and $\Delta \phi$ gets smaller. The distinction between the two phases has disappeared when their compressibility factors are equal. Extrapolating the linear plot of $\Delta \phi$ versus $\delta^{2}$ to $\Delta \phi=0$ gives the « critical » value $0.27_{5} \pm 0.01$ for the degree of polydispersity at which the freezing transition is supposed to disappear. The result is not particular to the procedure of Streett $e t$ al.; the set of tie-lines calculated by the alternative method of Hoover and Ross [9] occur at slightly higher pressures, but the general dependence on $\delta$ is much the same.

In the treatment presented here, we have assumed that the change in the phase diagram of the simulated colloid with increasing polydispersity is valid in the thermodynamic limit, and that the transition remains effectively first-order throughout. We find that the crystalline state is wholly disrupted once the distribution of particle sizes reaches a level of $c a .30 \%$ polydispersity. Irrespective of the nature of the particle-size distribution function or the interparticle forces, one can expect all highly polydisperse systems to exhibit glass formation in preference to crystallization.

\section{Acknowledgments.}

R.P. acknowledges S.E.R.C. and Unilever (Port Sunlight) for a Research Studentship under the C.A.S.E. scheme. 


\section{References}

[1] Dickinson, E., Chem. Phys. Lett. 57 (1978) 148.

[2] Dickinson, E., J. Chem. Soc., Faraday Trans. 275 (1979) 466.

[3] Dickinson, E., Faraday Discuss. R. Soc. Chem. 65 (1978) 127.

[4] Dickinson, E., Parker, R. and Lal, M., Chem. Phys. Lett. 79 (1981) 578.

[5] Parker, R., Ph. D. Thesis, University of Leeds, 1984.

[6] Hansen, J. P. and McDonald, I. R., Theory of Simple Liquids (Academic Press, London) 1976.

[7] Streett, W. B., Raveche, H. J. and Mountain, R. D., J. Chem. Phys. 61 (1974) 1960.

[8] Hoover, W. G. and ReE, F. H., J. Chem. Phys. 49 (1968) 3609.

[9] Hoover, W. G. and Ross, M., Contemp. Phys. 12 (1971) 339. 